\title{
Explaining socioeconomic differences in sickness absence: the Whitehall II study
}

\author{
Fiona North, S L Syme, Amanda Feeney, Jenny Head, Martin J Shipley, M G Marmot
}

\begin{abstract}
Objective-To describe and explain the socioeconomic gradient in sickness absence.

Design-Analysis of questionnaire and sickness absence data collected from the first phase of the Whitehall II study. Grade of employment was used as a measure of socioeconomic status.
\end{abstract}

Setting-20 civil service departments in London.

Subjects -6900 male and 3414 female civil servants aged 35-55 years.

Main outcome measures-Rates of short spells ( $\leqslant 7$ days) and long spells ( $>7$ days) of sickness absence.

Results-A strong inverse relation between grade of employment and sickness absence was evident. Men in the lowest grade had rates of short and long spells of absence $6 \cdot 1(95 \%$ confidence interval $5 \cdot 3$ to 6.9) and 6.1 (4.8 to $7 \cdot 9)$ times higher than those in the highest grade. For women the corresponding rate ratios were $3.0(2.3$ to 3.9$)$ and $4.2(2.5$ to 6.8$)$ respectively. Several risk factors were identified, including health related behaviours (smoking and frequent alcohol consumption), work characteristics (low levels of control, variety and use of skills, work pace, and support at work), low levels of job satisfaction, and adverse social circumstances outside work (financial difficulties and negative support). These risk factors accounted for about one third of the grade differences in sickness absence.

Conclusion-Large grade differences in sickness absence parallel socioeconomic differences in morbidity and mortality found in other studies. Identified risk factors accounted for a small proportion of the grade differences in sickness absence. More accurate measurement of the risk factors may explain some of the remaining differences in sickness absence but other factors, as yet unrecognised, are likely to be important.

and Social Medicine,

University of Otago,

Dunedin, New Zealand

Fiona North, research fellow

School of Public Health,

University of California,

Berkeley CA 94720, United

States

S L Syme, visiting professor

\section{Department of}

Epidemiology and Public

Health, University College and Middlesex School of

Medicine, University

College London, London

WC1E 6EA

Amanda Feeney, research

fellow

Jenny Head, lecturer in

statistics

Martin J Shipley, senior

lecturer in medical statistics

M G Marmot, professor of

epidemiology and public health

Correspondence to:

Professor Marmot.

BMF 1993;306:361-6

\section{Introduction}

An inverse relation between socioeconomic status and mortality has been documented in many studies, no matter how socioeconomic status has been measured. The challenge is to explain this relation. The Whitehall study of British civil servants, which started in 1967, ${ }^{1}$ showed a steep inverse association between social class (assessed by grade of employment) and mortality from a wide range of diseases. ${ }^{2}$ After 10 years of follow up those in the highest grades of employment had about one third the mortality of those in the lowest grades. This difference in mortality was only partly explained by differences in age, smoking, systolic blood pressure, height, and plasma cholesterol and blood glucose concentrations. ${ }^{2}$ The limited data available suggest there are also substantial socioeconomic differences in morbidity, ${ }^{34}$ but these differences remain largely unexplained. The Whitehall II study examined a new cohort of 10314 civil servants between 1985 and 1988 with the aim of explaining socioeconomic gradients in morbidity and mortality. In addition to the established risk factors included in the first Whitehall study, the second study has docu- mented differences by grade of employment in work characteristics, social support, and health related behaviours. $^{5}$

This paper seeks to describe and explain observed grade differences in sickness absence. Rates of sickness absence are used as a measure of morbidity. Like most measures of morbidity sickness absence is influenced by social and psychological factors as well as illness. Sickness absence is important as a measure of ill health; as a measure of use of health services; as a cause of lost productivity; and as an indicator of an employee's ability to cope with and maintain normal roles at work. The costs of sickness absence to the government and to industry are substantial. In the United Kingdom more than 370 million work days are lost each year owing to certified incapacity. ${ }^{6}$ A survey by the Confederation of British Industry in 1986 estimated that absence from work cost British industry at least $£ 5$ billion. ${ }^{7}$ Trends in sickness absence since the 1950 s suggest that these costs are likely to increase. ${ }^{89}$

\section{Subjects and methods} STUDY POPULATION

All non-industrial civil servants aged 35-55 working in the London offices of 20 departments were invited to participate in the study. The overall response rate was $73 \%$ ( $74 \%$ for men and $71 \%$ for women). The true response rate is likely to be higher, however, because around $4 \%$ of the civil servants on the lists provided by the civil service had moved before the study and were therefore not eligible for inclusion. The response rate varied by grade of employment, being $81 \%$ in the three higher grade categories (defined below) and $68 \%$ in the three lower categories. In total, 10314 civil servants participated, of whom $67 \%$ (6900) were men and 33\% (3414) were women.

Most participants (94\%) gave consent for follow up based on their sickness absence records. A small proportion of records $(5 \%)$ could not be identified. Sickness absence records of 9072 participants $(88 \%$ of the total sample) were examined over a mean period of 20 (range 0.3-39.6) months.

Information on grade of employment was obtained by asking participants to give their civil service grade at the time of the baseline survey. Changes in grade during the follow up period were not analysed. On the basis of salary, the civil service identifies 12 nonindustrial grades which, in order of decreasing salary, consist of seven "unified grades," senior executive officers, higher executive officers, executive officers, clerical officers, and clerical assistants and office support staff. Unified grade is used by the civil service to refer to the combination of administrative grades (previously known as permanent secretary, deputy secretary, under secretary, assistant secretary, senior principal, and principal) and professional or technical staff with equivalent salaries. Similarly, the remaining professional or technical staff are combined with administrative grades on the basis of salary. To obtain significant numbers we combined unified grades 1-6 into one category and clerical officers, clerical assistants, and office support staff into another category, thus producing six grade categories (table I). 
There was a steep increment in salaries between grade categories-from an annual salary in 1987 of $£ 18020$ $£ 62100$ in category 1 to $£ 3060-£ 6790$ in category 6 . However, most of the civil servants in the top category were at the lower end of the pay scale, with $82 \%$ of men and $83 \%$ of women in category 1 earning between $£ 18020$ and $£ 27065$. Differences in other socioeconomic indicators (education, housing tenure, car ownership, and father's occupation) by grade of employment have been described.

\section{BASELINE SURVEY}

Between November 1985 and March 1988 participants completed questionnaires and attended a screening examination. The questionnaire included personal details (age, sex, current grade of employment, ethnic group, marital status, years of full time education, highest level of education, partner's and father's occupation, housing tenure, and car ownership); health (self rated health over the past 12 months, presence of longstanding illness, and presence of recurring health problems based on questions used in the general household survey, ${ }^{10}$ and presence of psychiatric symptoms based on the 30 item general health questionnaire ${ }^{11}$ ); health related behaviours (current smoking habits, usual frequency of alcohol consumption in past 12 months, and amount of alcohol consumed in the past seven days); psychosocial work characteristics (assessed with a 67 item questionnaire based on the occupational strain model of job demands and decision latitude ${ }^{12}$ which included questions on control, variety and use of skills, and work pace as proposed by Karasek, ${ }^{12}$ support at work, ${ }^{13}$ and job satisfaction); social circumstances outside work (number of dependent children, social contact with relatives and friends, and personal difficulties such as financial problems); and types of social support (assessed by 15 self report questions on up to four nominated close friends or relatives). Three types of social support were confirmed by principal components analysis: confiding or emotional support, practical support, and negative aspects of support. Further details on the work and social support have been reported. ${ }^{5}$

\section{SICKNESS ABSENCE RECORDS}

Computerised sickness absence records to the end of March 1988 were obtained annually from the civil service pay centres. These records included the first and last dates of all absences and the reason for absence. For absences of seven calendar days or less (short spells), civil servants were able to complete their own certificate explaining their absence. For absences of more than seven calendar days (long spells), a medical certificate was required. Sickness absence records were checked for inconsistencies. Overlapping, consecutive, or duplicate spells of sickness absence were merged after taking account of weekends and public holidays. This affected less than $1 \%$ of all spells of sickness absence.

\section{STATISTICAL ANALYSIS}

Risk factors for sickness absence (or the size of their effect), may differ for short and long spells. Short spells (seven days or less) and long spells (more than seven days) of sickness absence were analysed separately. For each employee, the number of spells of sickness absence of each type was computed and the follow up period was measured in person years. Rates of sickness absence are expressed per 100 person years.

Age adjusted rates were calculated by direct standardisation with the total sample as the standard. The number of spells of sickness absence is a form of count data and therefore Poisson regression models were fitted to the data. ${ }^{115} \mathrm{We}$ assumed that for each participant the occurrence of short spells followed a Poisson distribution. The regression model for the $\mathrm{i}^{\text {th }}$ individual was

$\log \left(y_{i}\right)=\log \left(T_{i}\right)+\alpha+\beta_{1} x_{i 1}+\beta_{2} x_{i 2}+\ldots+\beta_{p} x_{i p}+\varepsilon_{i}$, where $y_{i}$ is the observed number of short spells of absence $T_{i}$ is the number of years of follow up, $\mathrm{x}_{\mathrm{il}} \ldots \mathrm{x}_{\mathrm{ip}}$ are the explanatory factors of interest, and $\varepsilon_{\mathrm{i}}$ is a Poisson error term.

Adjusted rate ratios and their 95\% confidence intervals were calculated for men and women separately by this method. The Poisson model implies that the variance, in the rates of sickness absence between individuals is equal to the expected rate of sickness absence. If rates of sickness absence vary between individuals after taking account of the explanatory factors this may lead to extra variation (overdispersion) relative to that predicted from the Poisson model. Considerable excess residual variation was found in the rate of sickness absence for short spells. A scale parameter was therefore estimated by dividing this residual variation (deviance) by the degrees of freedom and was used to adjust the width of the confidence intervals. ${ }^{1+15}$ This had no effect on the rate ratio estimates but the width of the confidence intervals was increased by about $50 \%$. For long spells of absence, the Poisson model was used but no overdispersion was detected.

Participants with incomplete data were excluded from the analyses including the missing variables. Several questions on health and social supports were added to a later version of the screening questionnaire. Consequently, the number of subjects varied when different explanatory variables were used. Comparison of age adjusted and fully adjusted rate ratios, however, were based only on subjects with no missing data. The overall trend in sickness absence rates across the grades was assessed by fitting a linear term for employment grade.

The regression models were fitted by using the statistical package GLIM. ${ }^{16}$ The error term was set as Poisson and the logarithm of person years at risk was declared as an offset. All other analyses were performed with the statistical package SAS.'

\section{Results}

Table II shows the total number of short and long spells and the overall rates of sickness absence for men and women. On average, men and women had 1.3 and $2 \cdot 1$ absences per person per year respectively for short

TABLE I-Grade distribution of civil servants in the Whitehall II study

\begin{tabular}{lccc}
\hline Category & Men & Women & Total $(\%)$ \\
\hline 1 & 1026 & 122 & $1148(11)$ \\
2 & 1627 & 264 & $1891(18)$ \\
3 & 1228 & 198 & $1426(14)$ \\
4 & 1496 & 480 & $1976(19)$ \\
5 & 881 & 660 & $1541(15)$ \\
6 & 642 & 1690 & $2332(23)$ \\
\hline Total & $6900(67 \%)$ & $3414(33 \%)$ & $10314(100)$ \\
\hline
\end{tabular}

*Grade category: 1=unified grade 1-6, 2=unified grade 7, 3=senior executive officer, $4=$ higher executive officer, $5=$ executive officer, $6=$ executive officer, $4=$ higher executive officer
clerical officer, assistant, office support staff. clerical officer, assistant, office support staff.
Categories $1-5$ include professional or technical equivalents.

TABLE II-Rate of sickness absence per 100 person years and number of short and long spells of absence

\begin{tabular}{lrrrrr}
\hline & \multicolumn{2}{c}{ Men } & & \multicolumn{2}{c}{ Women } \\
\cline { 2 - 3 } \cline { 5 - 6 } & $\begin{array}{c}\text { Rate/100 } \\
\text { person years }\end{array}$ & $\begin{array}{c}\text { No of } \\
\text { spells }\end{array}$ & & $\begin{array}{c}\text { Rate/100 } \\
\text { person years }\end{array}$ & $\begin{array}{l}\text { No of } \\
\text { spells }\end{array}$ \\
\hline Short absence* & 126.9 & 13208 & 208.5 & 9921 \\
Long absence $\dagger$ & 11.9 & 1233 & 30.2 & 1436 \\
\hline
\end{tabular}

$\star \leqslant 7$ days, no medical certificate required.

$\dagger>7$ days, medical certificate required. 
spells and $0 \cdot 1$ and 0.3 absences per person per year respectively for long spells.

Figures 1 and 2 show a strong inverse relation between grade of employment and rates of short and long spells of sickness absence adjusted for age. Except for women in the highest grade, there was a stepwise increase in rates of both short and long spells. For men, those in the lowest grade had rates of short and long spells of sickness absence $6 \cdot 1(95 \%$ confidence interval $5 \cdot 3$ to $6 \cdot 9)$ and $6 \cdot 1(4 \cdot 8$ to $7 \cdot 9)$ times higher than those in the highest grade, respectively. For women, the corresponding rate ratios were $3.0(2.3$ to 3.9$)$ and 4.2 $(2.5$ to 6.8$)$ respectively. The grade differences in sickness absence were present in all age groups.

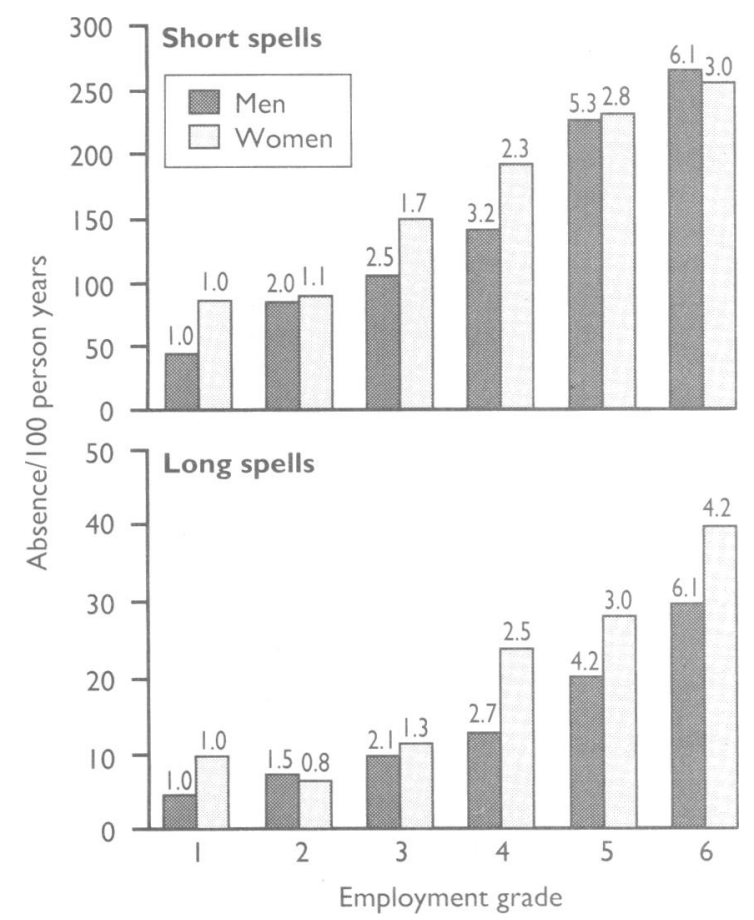

Age adjusted rates of short (top) and long (bottom) spells of sickness absence by sex and grade of employment. Age adjusted rate ratios are shown at top of each column

Table III shows the relation between other risk factors and sickness absence. Men and women who reported average or worse health in the past 12 months had $60 \%$ higher rates of short spells of absence and more than twice the rates of long spells compared with those who reported good health. Differences were also observed for other self reported measures of health, including the presence of recurring health problems, longstanding illness, and psychiatric symptoms. Par- ticipants who smoked also had higher rates of short and long spells of sickness absence compared with nonsmokers. The relation between alcohol consumption and sickness absence will be reported elsewhere. For men, there seemed to be a $U$ shaped relation between alcohol consumption and the rate of short spells of sickness absence, whereas for long spells only those who were frequent drinkers (more than once daily) had higher rates. For women, there was no clear relation between alcohol consumption and sickness absence. There was also a relation between ethnic group and sickness absence with higher rates of both short and long spells of absence in Asian men and women.

Psychosocial factors at work and outside work also predicted rates of sickness absence. Men and women who rated their jobs as low for control, variety and use of skills, pace, support at work, or satisfaction had higher rates of short and long spells of sickness absence compared with those who rated their jobs high for these characteristics. This was particularly striking for control and variety and use of skills. For example, men who reported low variety and use of skills had $72 \%$ and $82 \%$ higher rates of short and long spells of absence respectively, compared with those who reported high variety and use of skills. Similarly, participants who reported negative support from their closest friend or relative or financial difficulties had higher rates of sickness absence. Men who reported that talking with their closest friend or relative made things worse had $29 \%$ and $40 \%$ higher rates of short and long spells of absence, respectively, compared with those who reported positive support from this person. Men and women who reported great financial difficulties had about $40 \%$ higher rates of both short and long spells of absence compared with those who reported few financial difficulties.

Table IV shows the rates of sickness absence by grade after adjusting for the health related behaviours (smoking habits and frequency of alcohol consumption), ethnic group, work characteristics, and social circumstances outside work which predicted rates of sickness absence. The effect of the adjustment is greater in the lower than the higher grades, reflecting the fact that the mediating factors show progressive differences across the employment grades. When fitting a linear term for employment grade, the increasing trends in short spells of sickness absence across the grades were reduced by $27 \%$ for men and $40 \%$ for women from those seen before adjustment. Grade differences in long absences were reduced by $11 \%$ for men and by $36 \%$ for women after adjustment. Even after adjustment the differences between those at the top and those at the bottom remained substantial: among men, those in grade 6 had $3 \cdot 7$ and $6 \cdot 4$ times

TABLE III-Rate ratios * (95\% confidence intervals) of factors studied that predict sickness absence

\begin{tabular}{|c|c|c|c|c|c|c|}
\hline & \multicolumn{3}{|c|}{ Men } & \multicolumn{3}{|c|}{ Women } \\
\hline & $\begin{array}{c}\text { No of } \\
\text { subjects }\end{array}$ & Short spells & Long spells & $\begin{array}{l}\text { No of } \\
\text { subjects }\end{array}$ & Short spells & Long spells \\
\hline \multicolumn{7}{|l|}{ Health and health related behaviour: } \\
\hline Self rated health (average/worse $v$ good) & 6203 & $1.78(1.68$ to 1.87$)$ & $2 \cdot 36(2 \cdot 10$ to $2 \cdot 64)$ & 2845 & $1.56(1.47$ to 1.67$)$ & $2 \cdot 16(1.94$ to 2.39$)$ \\
\hline Recurring health problems (yes $v$ no) & 4774 & $1.43(1.33$ to 1.53$)$ & $1.70(1.44$ to 2.02$)$ & 2107 & $1.38(1.26$ to 1.52$)$ & $1.37(1.17$ to 1.61$)$ \\
\hline Longstanding illness (yes $v$ no) & 4783 & $1.34(1.26$ to 1.43$)$ & $1.96(1.70$ to 2.25$)$ & 2122 & $1.21(1.11$ to 1.30$)$ & $1.58(1.39$ to 1.80$)$ \\
\hline Psychiatric symptoms (yes $v$ no) & 6186 & $1.25(1.19$ to 1.33$)$ & $1.42(1.26$ to 1.60$)$ & 2807 & $1.13(1.06$ to 1.21$)$ & $1.13(1.01$ to 1.27$)$ \\
\hline Current smoking (yes $v$ no) & 6281 & $1.46(1.37$ to 1.55$)$ & $1.81(1.59$ to 2.06$)$ & 1434 & $1.09(1.02$ to 1.18$)$ & $1.37(1.22$ to 1.53$)$ \\
\hline \multicolumn{7}{|l|}{ Work characteristics: } \\
\hline Control (low $v$ high) & 6206 & $1.58(1.50$ to 1.67$)$ & $1.54(1.38$ to 1.72$)$ & 2799 & $1 \cdot 21(1.14$ to $1 \cdot 30)$ & $1.52(1.35$ to 1.71$)$ \\
\hline Variety and use of skills (low $v$ high) & 6204 & $1.72(1.63$ to 1.81$)$ & $1.82(1.63$ to 2.04$)$ & 2810 & $1.41(1.32$ to 1.51$)$ & $1.69(1.49$ to 1.91$)$ \\
\hline Support at work (low $v$ high) & 6205 & $1.26(1.19$ to 1.32$)$ & $1.07(0.96$ to 1.20$)$ & 2808 & $1.06(1.00$ to 1.13$)$ & $1.07(0.96$ to 1.19$)$ \\
\hline Work pace (low $v$ high) & 6205 & $1.43(1.36$ to 1.50$)$ & $1.27(1.13$ to 1.42$)$ & 2812 & $1.22(1.15$ to 1.30$)$ & $1.12(1.01$ to 1.25$)$ \\
\hline Job satisfaction (low $v$ high) & 6201 & $1.50(1.43$ to 1.58$)$ & $1.32(1.18$ to 1.48$)$ & 2800 & $1.22(1.14$ to 1.30$)$ & $1.07(0.96$ to 1.19$)$ \\
\hline \multicolumn{7}{|l|}{ Social circumstances outside work: } \\
\hline Negative aspects of support (high $v$ low) & 4669 & $1.29(1.21$ to 1.37$)$ & $1.40(1.22$ to 1.61$)$ & 2042 & $1 \cdot 12(1.04$ to $1 \cdot 21)$ & $1.05(0.92$ to 1.19$)$ \\
\hline $\begin{array}{l}\text { Difficulty paying bills (great/some } v \\
\text { slight/very little) }\end{array}$ & 4795 & $1.42(1.32$ to 1.51$)$ & $1.48(1.27$ to 1.72$)$ & 2132 & $1.28(1.18$ to 1.40$)$ & $1.55(1.35$ to 1.78$)$ \\
\hline
\end{tabular}

*Adjusted for age.

These explanatory variables were summarised by separating participants into two categories above and below the median. However, when adjusting for the effect of these factors on sickness absence, many of the factors were divided into three or four levels. 


\begin{tabular}{|c|c|c|c|c|}
\hline \multirow[b]{2}{*}{ Grade category } & \multicolumn{2}{|c|}{$\operatorname{Men}(n=4622)$} & \multicolumn{2}{|c|}{ Women $(n=1986)$} \\
\hline & $\begin{array}{l}\text { Age adjusted } \\
\text { rate ratio }\end{array}$ & $\begin{array}{l}\text { Fully adjusted } \\
\text { rate ratio* }\end{array}$ & $\begin{array}{l}\text { Age adjusted } \\
\text { rate ratio }\end{array}$ & $\begin{array}{l}\text { Fully adjusted } \\
\text { rate ratio* }\end{array}$ \\
\hline \multicolumn{5}{|l|}{ Short absences: } \\
\hline 1 & $1 \cdot 0$ & $1 \cdot 0$ & $1 \cdot 0$ & $1 \cdot 0$ \\
\hline 2 & $1.98(1.68$ to 2.33$)$ & $1.82(1.55$ to 2.15$)$ & $1.31(0.83$ to 2.08$)$ & $1.22(0.77$ to 1.94$)$ \\
\hline 3 & $2.15(1.83$ to 2.53$)$ & $1.86(1.58$ to 2.20$)$ & $1.89(1.21$ to 2.94$)$ & $1.76(1.13$ to 2.75$)$ \\
\hline 4 & $2.85(2.43$ to 3.33$)$ & $2.32(1.97$ to 2.73$)$ & $2.32(1.53$ to 3.52$)$ & $1.99(1.31$ to 3.04$)$ \\
\hline 5 & $4 \cdot 80(4 \cdot 10$ to $5 \cdot 61)$ & $3.43(2.95$ to 4.13$)$ & $2.62(1.74$ to 3.94$)$ & $2.07(1.36$ to 3.16$)$ \\
\hline 6 & $5.61(4.78$ to 6.58$)$ & $3.69(3.07$ to 4.43$)$ & $2.93(1.96$ to 4.39$)$ & $2.11(1.38$ to 3.22$)$ \\
\hline \multicolumn{5}{|l|}{ Long absences: } \\
\hline 1 & $1 \cdot 0$ & $1 \cdot 0$ & $1 \cdot 0$ & $1 \cdot 0$ \\
\hline 2 & $1.73(1.19$ to 2.50$)$ & $1.75(1.21$ to 2.54$)$ & $1.01(0.43$ to 2.38$)$ & $1.08(0.44$ to 2.64$)$ \\
\hline 3 & $2.14(1.49$ to 3.08$)$ & $2.12(1.46$ to 3.07$)$ & $1.22(0.53$ to 2.81$)$ & $1.22(0.51$ to 2.90$)$ \\
\hline 4 & $3.02(2.13$ to 4.30$)$ & $2.89(2.00$ to 4.16$)$ & $2.07(0.98$ to 4.36$)$ & $1.87(0.86$ to 4.09$)$ \\
\hline 5 & $4.36(3.07$ to 6.21$)$ & $3.95(2.70$ to 5.78$)$ & $2.96(1.43$ to 6.14$)$ & $2.33(1.07$ to 5.04$)$ \\
\hline 6 & $7.35(5.19$ to $10 \cdot 40)$ & $6.44(4.31$ to 9.64$)$ & $3.65(1.78$ to 7.49$)$ & $2.55(1.18$ to 5.53$)$ \\
\hline
\end{tabular}

*Adjusted rate ratios are controlled for age, smoking, frequency of alcohol consumption, work characteristics (control, variety and use of skills, support at work, work pace, and job satisfaction), social circumstances outside work (negative support and difficulty paying bills), and demographic factors (ethnic group).

higher rates of sickness absence than those in grade 1 for short and long spells respectively. Among women, the corresponding ratios were $2 \cdot 1$ and $2 \cdot 6$ respectively. Adjustment for work characteristics and social circumstances outside work alone reduced the trends in short absences across the grades by $26 \%$ for men and $35 \%$ for women. The trends in long absences across the grades, however, were reduced by only $5 \%$ for men and $15 \%$ for women. Other risk factors examined were the frequency of social contact, father's social class, age joined civil service, number of children, attendance at religious services, and physical activity. None of these was a confounder or mediator of the relation between grade and sickness absence.

\section{Discussion}

Our findings are consistent with reports of higher rates of sickness absence among less skilled nonmanual or manual employees. ${ }^{18-24}$ Despite the consistency of the socioeconomic differences in sickness absence no studies have examined possible explanations for them. We found striking gradients in both short and long spells of sickness absence, with higher rates among employees with lower status. Moreover, the gradients were observed even among managerial and executive staff (grade categories 1-4).

We have used sickness absence as a measure of morbidity. Several findings support this interpretation. Similar grade differences in both short and long spells of sickness absence were observed. This suggests that the grade differences cannot be attributed to employees in the lower grades being more likely to take the odd day off or to those in the higher grades staying at work despite minor illness. Perceived health status was a strong predictor of rates of both short, and to a greater extent, long spells of sickness absence. Earlier studies have reported that perceived health status predicts subsequent mortality. ${ }^{25}{ }^{26}$ As further follow up data accumulate we will be able to examine whether sickness absence predicts serious morbidity and mortality in the Whitehall II study. Psychological and social factors predicted rates of short, and to a lesser extent, long spelis of absence. Most measures of morbidity which assess functional impairment, of which sickness absence is one, are influenced by factors other than health. The decision to take time off work or to return to work is complicated and will vary between individuals and at different times.

\section{EXPLANATIONS FOR DIFFERENCES}

Substantial differences in sickness absence by grade remained even after adjustment for a wide range of risk factors. This is consistent with several earlier studies which have attempted to explain socioeconomic differences in morbidity and mortality by differences among subjects in known or suspected risk factors. ${ }^{27}{ }^{28}$ In the first Whitehall study risk factors such as age, smoking, systolic blood pressure, height, and plasma cholesterol and blood glucose concentration accounted for about a fifth of the twofold to threefold grade differences in mortality from coronary heart disease. ${ }^{2}$ Similarly in the British regional heart study age, smoking, systolic blood pressure, and serum cholesterol concentration accounted for less than a sixth of the 1.5 fold social class differences in coronary heart disease morbidity and mortality. ${ }^{27}$ In the Alameda county study age, sex, race, socioeconomic factors (income, education, employment), health status, health related behaviours (smoking, alcohol consumption, physical activity, body mass index, and sleep patterns), access to medical care, social support, and psychological factors accounted for less than a sixth of the $1 \cdot 7$-fold difference in mortality from all causes between poor and more affluent areas. ${ }^{28}$ These studies suggest that, even when recognised risk factors exist, as for coronary heart disease, and differences in subjects' risk factors are taken into account, the relation between socioeconomic status and health cannot be fully explained.

In the Whitehall II study, differences between subjects in health, health related behaviours, psychosocial aspects of the work environment, and social circumstances outside work accounted for about a third of the threefold to sixfold differences in sickness absence by grade. This still leaves a large proportion of the variation unexplained.

Several explanations for the persistent grade differences in sickness absence need to be considered. Firstly, the grade differences in sickness absence may have been spurious. Anecdotal evidence suggests that managers and professional employees are more likely to be absent from work without record than employees with lower status. There was general agreement among senior civil servants that the recording of sickness absence may be incomplete among a small proportion of participants in grade category 1, but this was unlikely to occur in other categories. Although it was not possible to evaluate the completeness of the sickness absence records directly, the records were used for pay purposes and were therefore likely to be complete for most participants. Incomplete recording may partly account for the low rates of sickness absence among those in the highest grades, but it is unlikely to explain the large differences between other grades.

Secondly, several explanatory variables were difficult to measure and were only measured at one point in time. The work characteristics and social support were more likely to have been misclassified than some of the other explanatory variables. This would result in underestimation of their effects and incomplete adjustment of the grade differences in sickness absence. ${ }^{29}$ The risk factors therefore probably accounted for a greater proportion of the grade differences in sickness absence than was observed in the multivariate analysis.

Thirdly, factors which were not measured might be important. Risk factors specific to the individual such as work ethic or commitment to the organisation need to be considered. Alternatively, risk factors at the group level, such as attitudes about acceptable levels of sickness absence, may be important. Several papers have discussed the concept of absence cultures. ${ }^{3031}$ Absence cultures refer to shared attitudes about the level of sickness absence which is tolerated by groups within organisations. Over 40 years ago, Hill and Trist suggested that on joining an organisation employees observe the formal and informal responses of the organisation towards different levels of sickness absence and adopt levels of sickness absence that reflect these observations. ${ }^{32}$ While these factors may 
influence rates of short spells of sickness absence, they are less likely to influence rates of long spells. It is more likely that other factors, as yet unrecognised, are important.

\section{APPLICATION OUTSIDE CIVIL SERVICE}

To what extent can these findings from the British civil service be generalised to other occupational groups? The study population consisted of a representative sample of non-industrial civil servants in a diverse range of departments in London. At the time of the study, about 75000 civil servants from a total of about 700000 were based in central London. The organisational culture of the civil service may differ from organisational cultures in the private sector but is likely to be similar to other office based white collar organisations in the public sector. The civil service has a generous sickness absence policy, and civil servants receive full pay for sickness absence of up to six months in any 12 months. Employees with prolonged or repeated sickness absence are referred to the occupational health service. The civil service tends to offer good job security. In this study, the mean length of service was 19 years for men and 15 years for women. However, since 1985 several participating departments have undergone large organisational changes which are likely to affect job security. Factors which are more common in large urban areas, such as complex commuter travel, have been shown to predict rates of sickness absence. ${ }^{23} \mathrm{We}$ did not assess travel to work in detail. However, participants' living circumstancesfor example, housing tenure and car ownership-were similar to those in comparable socioeconomic groups in national surveys (Department of Employment, personal communication 1987). ${ }^{3}$ Despite these considerations, overall rates of sickness absence in the civil service are similar to, or lower than, those observed in other British organisations. ${ }^{33}$

The civil service had several advantages for a study of this type. It was possible to examine sickness absence within an organisation with a single sickness absence policy. Turnover was low so there was minimal loss to follow up. Successful collaboration during the first Whitehall study made it easier to agree on potentially difficult issues such as obtaining sickness absence records from the pay centres. Compared with studies based on the registrar general's classification of socioeconomic status, the occupations within grade categories in the civil service were relatively homogeneous. However, between grade categories there were striking differentials in income and other measures of socioeconomic status. ${ }^{5}$ Finally, it is possible to compare the findings for men and women with similar occupational status. There were interesting differences in the sociodemographic background of men and women in the same grades-men in the higher grades were more likely to be married and have dependent children than women, whereas the opposite pattern was observed in the lower grades. Explanations for sex differences in sickness absence are being analysed and will be reported elsewhere.

The implications of these findings need to be considered. Sickness absence is a big problem both in terms of lost productivity and cost and in terms of employees' wellbeing. Some of the risk factors identified, such as the work characteristics, could be modified to reduce sickness absence. However, substantial grade differences in sickness absence persist after taking account of a wide range of risk factors. These large differences in sickness absence rates among employment grades categories parallel differences seen in morbidity and mortality in other studies. None of these studies have been able to "explain" fully the observed gradients. One possible explanation for this difficulty is that research rarely considers the broad range of causal factors that is probably necessary. A dominant tradition in public health research has been the risk factor approach, emphasising such phenomena as cigarette smoking, obesity, and blood pressure. Another tradition, focusing on psychosocial factors, typically de-emphasises or neglects the role of these established risk factors. We are now collecting both types of data, which will allow us to examine their joint effects on sickness absence and mortality. However, it is also likely that other factors, as yet unrecognised, are important. Socioeconomic differences in sickness absence warrant further investigation.

We thank all participating civil service departments and their welfare, personnel, and establishment officers; the Civil Service Occupational Health Service, Dr George Sorrie, and Dr Adrian Semmence; the Civil Service Central Monitoring Service and Dr Frank O'Hara; the Council of Civil Service Unions; and all participating civil servants. We also thank Alan Harding for computer support; screening coordinator Julie Moore; and Aleks Macheta for data management.

The study was supported by the Medical Research Council, Health and Safety Executive, Civil Service Occupational Health Service, National Heart Lung and Blood Institute (2 RO1 HL36310-04), Agency for Health Care Policy Research (5 RO1 HS06516), Ontario Workers' Compensation Institute, and The John D and Catherine $\mathrm{T}$ MacArthur Foundation Research Network on Successful Midlife Development. We thank members of the Canadian Institute of Advanced Research and the research division of the Ontario Workers' Compensation Institute for their critical discussion of this research. Fiona North was supported by the Medical Research Council of New Zealand and National Heart Foundation of New Zealand.

1 Reid DD, Brett GZ, Hamilton PJS, Jarrett RJ, Keen H, Rose G. Cardiorespiratory disease and diabetes among middle-aged male civil servants. Lancet 1974;i:469-73.

2 Marmot MG, Shipley MJ, Rose G. Inequalities in death-specific explanations of a general pattern? Lancet 1984;i:1003-6.

3 Office of Population Censuses and Surveys. General household survey 1987 London: HMSO, 1989.

4 Blaxter $\mathrm{M}$. Evidence on inequality in health from a national survey. Lancet 1987;ii:30-3.

5 Marmot MG, Davey Smith G, Stansfeld S, Patel C, North F, Head J, et al. Health inequalities among British civil servants: the Whitehall II study. Lancet 1991;337:1387-93.

6 Central Statistical Office. Regional trends 20. London: HMSO, 1985.

7 Confederation of British Industry: Absence from work: a survey of absence and non-attendance. London: CBI, 1987.

8 Morris JN. Capacity and incapacity for work: some recent history. Proc $R$ Soc Med 1965;58:821-5.

9 Alderson MR. An introduction to epidemiology. London: MacMillan Press, 1983.

10 Office of Population Censuses and Surveys. General household survey 1977. London: HMSO, 1979.

11 Goldberg DP. The detection of psychiatric illness by questionnaire. Oxford: Oxford University Press, 1972. (Maudsley Monograph No 21.)

12 Karasek R, Baker D, Marxer F, Ahlbom A, Theorell T. Job decision latitude, job demands and cardiovascular disease: a prospective study of Swedish men. Am f Public Health 1981;71:694-705.

13 Johnson JV, Hall EM. Job strain, work place social support, and cardiovascular disease: a cross-sectional study of a random sample of the Swedish working population. Am $\mathcal{F}$ Public Health 1988;78:1336-42.

14 McCullagh P, Nelder JA. Generalised linear models. London: Chapman and Hall, 1983:127-40

15 Aitken M, Anderson D, Francis B, Hinde J. Statistical modelling in GLIM. New York: Oxford University Press, 1989:217-25.

6 Numerical Algorithms Group. The GLIM system release 3.77 manual. 2nd ed. Oxford: Numerical Algorithms Group, 1987.

7 SAS. SAS user's guide, version 5. Cary NC: SAS Institute, 1985.

18 Alderson MR. Data on sickness absence in some recent publications of the Ministry of Pensions and National Insurance. Br f Prev Soc Med 1967;21: Minis.

19 Office of Population Censuses and Surveys. General household survey, 1976. London: HMSO, 1978.

20 Soderfelt B, Danermark B, Laarson S. Social class and sickness absence. Scand F Soc Med 1987;15:11-7.

21 Taylor PH. Occupational and regional associations of death, disablement and sickness absence among Post Office staff 1972-75. Br $\mathrm{f}$ Ind Med 1976;33 230-5.

22 Thomson D. Sickness absence in the civil service. Proc $R$ Soc Med 1972;65: $572-7$.

23 Taylor PJ, Pocock SJ. Commuter travel and sickness absence of London office workers. Br 7 Prev Soc Med 1972;26:165-72.

24 Pines A, Skulkeo K, Pollak E, Peritz E, Steif J. Rates of sickness absenteeism among employees in a modern hospital: the role of demographic and occupational factors. Br f Ind Med 1985;42:326-35.

25 Kaplan GA, Camacho T. Perceived health and mortality: a nine year follow-up of the human population laboratory cohort. Am $f$ Epidemiol 1983;117 of the hum.

26 Idler EL, Angel RJ. Self-rated health and mortality in the NHANES-I epidemiologic follow-up study. Am $\mathcal{Y}$ Public Health 1990;80:446-52.

27 Pocock SJ, Shaper AG, Cook DG, Phillips AN, Walker M. Social class 
differences in ischaemic heart disease in British men. Lancet 1987;ii: 197-201.

28 Haan M, Kaplan GA, Camacho T. Poverty and health: prospective evidence from the Alameda county study. Am $\mathcal{F}$ Epidemiol 1987;125:989-98.

29 Greenland S. The effect of misclassification in the presence of covariates. Am 7 Epidemiol 1980;112:564-9.

30 Johns $\mathrm{G}$, Nicholson $\mathrm{N}$. The meaning of absence: new strategies for theory and research. Research in Organizational Behaviour 1982;4:127-72.
31 Nicholson N, Johns G. The absence culture and the psychological contractwho's in control of absence. Academy Management Review 1985;10:397-407. 32 Hill JM, Trist EL. Changes in accidents and other absences with length of service. Human Relations 1955;8:121-52.

33 Civil Service Medical Advisory Service. The health of the civil service. London: HMSO, 1985.
Department of Social and Preventive Medicine, Medical Schopl, University 6f Queensland. Hersten, Queensland, Australia) 4006 RP Glasziou, senior lecturer in clinical epidemiology

\section{Department of Public} Health, University of Sydney, New South Wales, Australia 2006

D E M Mackerras, lecturer

in nutritional epidemiology

Correspondence to: Dr Glasziou.

$B M F$ 1993;306:366-70

\title{
Vitamin A supplementation in infectious diseases: a meta-analysis $/$
}

\author{
P P Glasziou, D E M[Mackerras
}

\section{Abstract}

Objective-To study the effect of vitamin A supplementation on morbidity and mortality from infectious disease.

Design-A meta-analysis aimed at identifying and combining mortality and morbidity data from all randomised controlled trials of vitamin $A$.

Results-Of 20 controlled trials identified, 12 trials were randomised trials and provided "intention to treat" data: six community trials in developing countries, three in children admitted to hospital with measles, and three in very low birth weight infants. Combined results for community studies suggest a reduction of $30 \%(95 \%$ confidence interval $21 \%$ to $38 \%$; two tailed $p<0.0000001$ ) in all cause mortality. Analysis of cause specific mortality showed a reduction in deaths from diarrhoeal disease (in community studies) by $39 \%$ ( $24 \%$ to $50 \%$; two tailed p $<0.00001)$; from respiratory disease (in measles studies) by $70 \%$ $(15 \%$ to $90 \%$; two tailed $p=0.02)$; and from other causes of death (in community studies) by $34 \%$ (15\% to $48 \%$; two tailed $p=0.001$ ). Reductions in morbidity were consistent with the findings for mortality, but fewer data were available.

Conclusions-Adequate supply of vitamin A, either through supplementation or adequate diet, has a major role in preventing morbidity and mortality in children in developing countries. In developed countries vitamin A may also have a role in those with life threatening infections such as measles and those who may have a relative deficiency, such as premature infants.

\section{Introduction}

In 1928 Green and Mellanby noted that though vitamin $\mathrm{A}$ was then known as the growth promoting vitamin, evidence from animal studies showed it was also an anti-infective vitamin. ${ }^{\prime}$ Four years later, Ellison reported a controlled trial of 600 English children hospitalised with measles which showed that cod liver oil reduced mortality by $58 \%{ }^{2}$ But the role of vitamin $\mathrm{A}$ in preventing xerophthalmic blindness, combined with the discovery of antibiotics, overshadowed its possible anti-infective role.

In 1986 Sommer et al reported a seminal randomised trial showing a $34 \%$ reduction in the all cause mortality of preschool Indonesian children without florid signs of deficiency as a result of two 200000 IU doses of vitamin A given six months apart. ${ }^{3}$ Several months later Barclay et al reported a 50\% reduction in mortality in children hospitalised with measles, although this result was not statistically significant. ${ }^{4} \mathrm{~A}$ recent large randomised study in India found no such effect but rather an apparent slight excess of deaths in the supplemented group. ${ }^{56}$ In the subsequent exchange of letters it was asserted that there is no evidence that high doses of vitamin A reduce the rate of infection and, as a mechanism was lacking, the effect on mortality was equally suspect. $^{78}$
Given the magnitude of the apparent benefit and the simplicity and inexpensiveness of the intervention, clarification of the role of vitamin A is of considerable importance. We therefore performed a meta-analysis of the available randomised trials of vitamin A supplementation, looking at total mortality, cause specific mortality, and morbidity from infectious diseases.

\section{Methods}

IDENTIFICATION OF TRIALS

We aimed to examine and combine all randomised controlled trials of vitamin A supplementation for the prevention of death or morbidity from infectious disease, in particular respiratory and gastrointestinal disease. Two methods were used to locate primary research data. Medline was searched independently by one of the authors, a research assistant, and a librarian for the years 1969 to 1992 , using combinations of the following key words: vitamin A, respiratory disease, diarrhoea, random allocation, and clinical trial. In addition, the references of the available primary studies, review articles, and editorials were checked to identify references not found in the Medline searches.

A report was dropped from further analysis if the study did not include concurrent controls or contained no original data or if the report did not address mortality, respiratory disease, or diarrhoea. Several trials that looked at cancer prevention were not considered within the scope of this analysis. On the basis of these initial broad criteria, clearly irrelevant articles were discarded after consideration by a single reviewer.

The methods sections of the potentially relevant reports were then extracted with reference to results and identifying material (authors, title, journal, institution, and country of origin) removed. These methods sections were assessed independently by two reviewers with respect to randomisation (individual, cluster, or none), use of a placebo, loss to follow up, measuring outcome blind to treatment assigned. Papers that did not include a control group, were not randomised, did not allow calculation of "intention to treat" results, or did not collect information on mortality or the incidence of respiratory or gastrointestinal infection were excluded.

\section{STATISTICAL METHODS}

We extracted from each trial data for an analysis by intention to treat-that is, an analysis that retains all individuals within the group to which they were randomised, regardless of compliance. Regrouping of data was necessary for one trial: table III of that paper was used to calculate the deaths in each group irrespective of the dose of vitamin A actually received. ${ }^{5}$

The Mantel-Haenszel estimate of the overall odds ratio and its variance were calculated from a set of studies by the Robins-Breslow-Greenland method. ${ }^{9}$ To test whether the variation between studies was explicable by chance, the Breslow and Day approxima- 